Strain, M. J., Lacava, C., Meriggi, L., Cristiani, I., and Sorel, M. (2015) Tunable Q-factor silicon micro-ring resonators for ultra-low power parametric processes. Optics Letters, 40(7), pp. 1274-1277.

Copyright (C) 2015 Optical Society of America

A copy can be downloaded for personal non-commercial research or study, without prior permission or charge

Content must not be changed in any way or reproduced in any format or medium without the formal permission of the copyright holder(s)

When referring to this work, full bibliographic details must be given

http://eprints.gla.ac.uk/103594

Deposited on: 01 May 2015

Enlighten - Research publications by members of the University of Glasgow http://eprints.gla.ac.uk 


\title{
Tunable Q-factor silicon micro-ring resonators for ultra-low power parametric processes
}

\author{
Michael J. Strain ${ }^{1,2}$, Cosimo Lacava ${ }^{3, \dagger}$, Laura Meriggi ${ }^{2}$, Ilaria Cristiani ${ }^{3}$, Marc Sorel $^{2}$ \\ ${ }^{1}$ Institute of Photonics, Department of Physics, University of Strathclyde, Glasgow, G4 ONW, UK \\ ${ }^{2}$ School of Engineering, University of Glasgow, Glasgow, G12 8LT, UK \\ ${ }^{3}$ Department of Electrical, Computer and Biomedical Engineering, University of Pavia, I-27100 Pavia, Italy \\ ${ }^{\dagger}$ Currently, Optoelectronics Research Centre, University of Southampton, Southampton SO17 1BJ, U.K. \\ ${ }^{*}$ Corresponding author: michael.strain@strath.ac.uk
}

Compiled March 2, 2015

\begin{abstract}
A compact silicon ring resonator is demonstrated that allows simple electrical tuning of the ring coupling coefficient and Q-factor and therefore the resonant enhancement of on-chip non-linear optical processes. Fabrication induced variation in designed coupling fraction, crucial in the resonator performance, can be overcome using this post-fabrication trimming technique. Tuning of the micro-ring resonator across the critical coupling point is demonstrated, exhibiting a Q-factor tunable between 9000 and 96000 . Consequently, resonantly enhanced Four Wave Mixing shows tunable efficiency between $-40 d B$ and $-16.3 d B$ at an ultra-low on-chip pump power of $0.7 \mathrm{~mW}$. (C) 2015 Optical Society of America
\end{abstract}

OCIS codes: $000.0000,999.9999$.

Optical parametric processes such as Four Wave Mixing (FWM) [1], are the foundation of all-optical switching $[2,3]$, multi-wavelength broadcasting [4] and correlated/entangled photon pair generation for quantum optical systems [5,6]. Resonant enhancement of these nonlinear effects allows their realisation with low optical pump powers $[1,5,7]$ and therefore the possibility of their inclusion in multi-component Photonic Integrated Circuits (PICs). In particular the Silicon-on-Insulator (SOI) material platform has proven extremely attractive for the realisation of PIC technologies, in part due to fabrication processes that are compatible with electronics manufacturing and low wafer costs. The large refractive index contrast of these waveguides is also advantageous, allowing for confinement of the optical mode to waveguide cross-sections with sub-micron dimensions, and therefore extremely compact devices can be produced with micron-scale footprints. Furthermore, the strong confinement of the optical mode alongside the significant $\chi^{(3)}$ nonlinearity of silicon [8] make this waveguide system an excellent platform for non-linear optical processes. The efficiency of these non-linear processes can be greatly enhanced by harnessing the resonant effect of compact devices afforded in the SOI platform. However, although resonator devices with low mode volumes, such as photonic crystal cavities, are attractive, they are difficult to design and fabricate [9]. Alternatively micro-ring resonators are relatively straightforward to produce, with devices already demonstrated in linear optical applications from signal filtering and routing in telecommunications systems $[10,11]$ to biosensing $[10,12]$. Nevertheless, fabrication tolerances still play a role in the performance of these resonators. To produce the maximum non-linear resonant enhancement, and therefore secondary effects such as photon-pair generation rate [13], the ring resonator should be operated at its critical coupling point, i.e. the condition where the round-trip loss factor of the ring is equal to the self-coupling coefficient [14]. For low loss silicon devices this condition requires low values of power coupling between the bus waveguide and the ring in the order of a few percent. The potential nm-scale variations in device dimensions due to fabrication process tolerance can then have a significant effect on the power coupling and hence the resonantly enhanced optical processes. For example, a typical SOI ring resonator with a $214 \mu \mathrm{m}$ path length (radius $R=15 \mu \mathrm{m}$, coupler length $\left.L_{c}=60 \mu \mathrm{m}\right)$ can be considered. A schematic of the device is shown as an inset to Fig.1. Ring propagation and coupler losses of $0.9 \mathrm{dBcm}^{-1}$ and $0.06 \mathrm{~dB}$ respectively, require a power cross-coupling coefficient, $\kappa$, of 0.018 to meet the critical coupling condition. Fig.1 shows a calculation of the raised Field Enhancement (FE) factor $\left(F E^{8}\right)$ [15] for FWM, due to such a ring resonator as a function of $\kappa$. The FE factor drops off very quickly in the under-coupled regime of the ring (i.e. $\kappa<\kappa_{\text {crit }}$ ). In the over-coupled regime $\left(\kappa>\kappa_{\text {crit }}\right)$ the FE factor also reduces, but with a shallower gradient. It is clear that a variation of only a few percent in the ring cross-coupling coefficient can lead to a significant decrease in the resonantly enhanced FWM efficiency. Such changes are easily generated by typical fabrication tolerances for evanescently coupled micro-ring resonators, and therefore large numbers of devices need to be fabricated to ensure that critical coupling is statistically likely to be achieved for a single device in the ensemble. This is clearly undesirable if such resonators are to be used in larger PICs. Resonators that can be tuned post-fabrication have been reported, including devices with MEMs actuated [16] and thermally controlled Mach-Zehnder (MZ) coupling sections [17]. However, these devices exhibit large footprints and in the case of the MEMs actuators, the fabrication of waveguide membranes creates structures that are ex- 


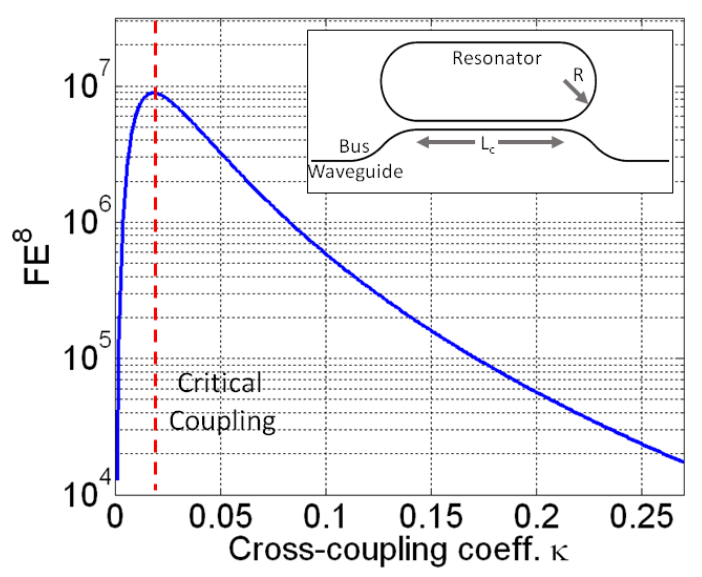

Fig. 1. Calculation of the raised Field Enhancement factor $\left(F E^{8}\right)$ for a silicon micro-ring resonator. (Inset: schematic of the ring resonator device.)

tremely sensitive to environmental factors. In this work an alternative, tunable evanescent field coupler [18] is used to control the operating point of the ring filters, as shown in Fig.2, allowing tuning of the resultant FWM efficiency.

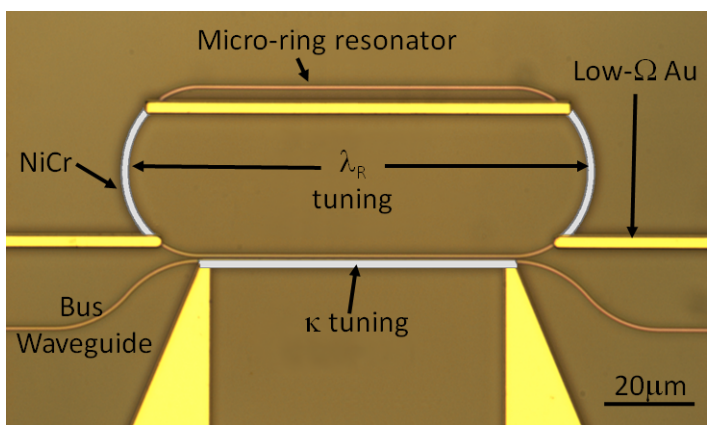

Fig. 2. Optical micrograph of a silicon micro-ring resonator with tunable cross-coupling coefficient $(\kappa)$ and central wavelength $\left(\lambda_{R}\right)$. NiCr elements are in false colour to contrast with low resistance tracks.

The devices presented in this work were fabricated on a SOI wafer with a $220 \mathrm{~nm}$ thick core layer on a $2 \mu \mathrm{m}$ buried oxide under-cladding. The waveguides were designed to be $500 \mathrm{~nm}$ in width and were clad with a $900 \mathrm{~nm}$ thick $\mathrm{SiO}_{2}$ layer. Transmission measurements on simple straight waveguides were performed to characterise the waveguide propagation losses and wavelength dependent group index [19]. Average propagation losses in these waveguides were $0.9 \mathrm{dBcm}-1$. The waveguide dispersion in the $1500-1600 \mathrm{~nm}$ was measured to be anomalous, with $D=600 \mathrm{ps} .\left(\mathrm{nm}^{-1} \mathrm{~km}^{-1}\right)$. The device includes resistive heating elements for control of the filter central wavelengths and cross-coupling coefficient. The racetrack geometry is used to allow better control over the as-fabricated coupling coefficient, with a coupler length of $60 \mu \mathrm{m}$ and bend radii of $15 \mu \mathrm{m}$. The devices were defined with e-beam lithography into HSQ re- sist and the pattern transferred into the silicon layer using reactive ion etching. Low resistance tracks were patterned in $\mathrm{Au}$, with the resistive heating elements defined as $50 \mathrm{~nm}$ thick, $900 \mathrm{~nm}$ wide $\mathrm{NiCr}$ lines. Inverse silicon waveguide tapers embedded in SU8 polymer waveguides were fabricated at the device facets to reduce coupling losses to the chip. The $\kappa$ tuning element is an asymmetrically placed resistive heater fabricated above the evanescent field coupler as detailed in [18]. The coupler was designed to be over-coupled, so that when a thermal gradient between the two waveguides of the evanescent field coupler is generated by the heating element, their effective refractive indices can be detuned and the coupler dephased. This dephasing results in a lower cross-coupling coefficient to the ring and hence tuning towards the critical coupling point. The thermal tuning of the coupler section also induces an optical path length change of the ring, shifting the resonance wavelength. The second heater element on the ring bends is designed to allow direct tuning of the resonance wavelength over a full Free Spectral Range (FSR), therefore allowing any change in wavelength due to coupler tuning to be compensated for.

The micro-ring device was designed to operate in the strongly over-coupled regime to demonstrate the wide tuning range of the coupler and access to the critical coupling point from a largely detuned starting point. The device had a bus-ring waveguide spacing of $350 \mathrm{~nm}$, producing an as fabricated coupling fraction of around 0.2. Fig. 3 shows the transmission spectra of the device, with dissipated power on the resistive heater as a parameter. All transmission spectra were measured at low input power levels to avoid thermal bistability effects in the swept-laser measurements. By curve fitting the trans-

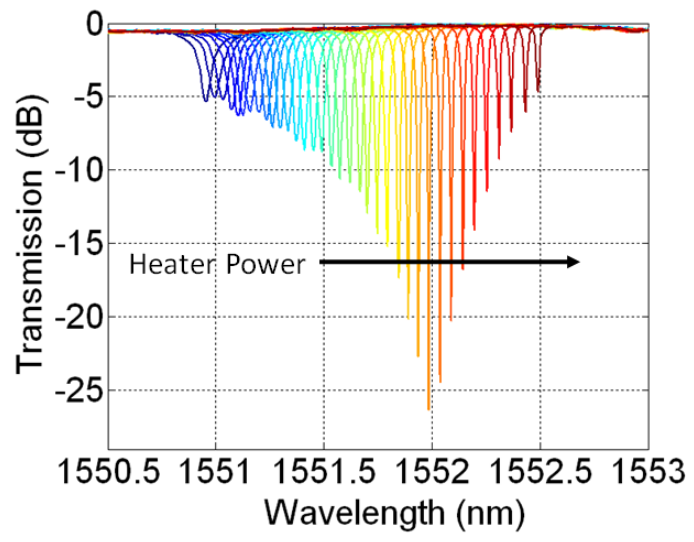

Fig. 3. Transmission spectra for a micro-ring resonator with varying power dissipated on the $\kappa$ tuning heater.

mission spectra to a transfer matrix model of an all-pass ring resonator [20] the coupling coefficient can be extracted as a free parameter. Fig.4(a) and (b) show the relationship between the dissipated power on the heater and the power cross-coupling coefficient $\kappa$ and resonator loaded Q-factor, respectively, where the Q-factor is calculated from the measured linewidth of the resonance. 
The dissipated power on the heater required to reach the critical coupling point is shown as dashed lines on the plots. The ring coupling coefficient was successfully

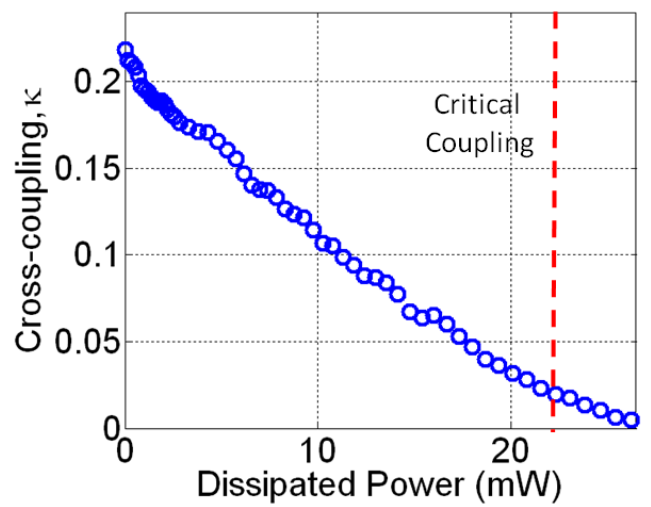

(a)

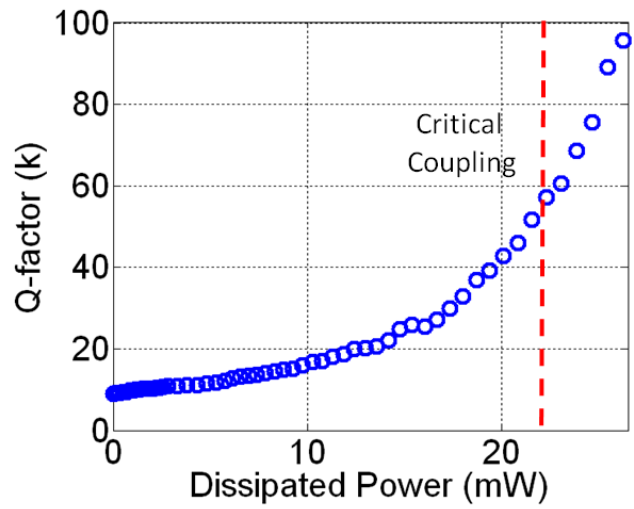

(b)

Fig. 4. (a) Ring cross-coupling coefficient $\kappa$ and (b) loaded Q-factor, as a function of power dissipated in the heater.

tuned from the as-fabricated value of $\kappa=0.22$, across the critical coupling point $(\kappa=0.018)$, down to a value of $\kappa=0.005$. Further tuning of the coupling coefficient was limited to avoid damage to the heater device. This tuning in cross-coupling coefficient in turn produced a Q-factor that was quasi-continuously tunable by over an order of magnitude between 9000 and 96000. The loaded Q-factor of the device at its critical coupling point was 57000 .

FWM experiments were carried out by coupling two tunable laser sources through polarisation controls and a $3 d B$ coupler to the lensed fibre input to the device. One laser source was amplified using an EDFA to act as the pump source. Coupling losses were $6 d B$ at each facet between the lensed fibre and SU8 waveguides on the chip. All references to injection power in this work refer to the on-chip pump power in the silicon bus waveguide. The pump power for the measurements presented below was $0.7 \mathrm{~mW}$. This corresponds to the power level at which the efficiency of the FWM process is saturated by non-linear losses. The pump laser wavelength was aligned to a resonance of the ring around $1550 \mathrm{~nm}$ and the signal laser aligned to the adjacent resonance on the longer wavelength side of the pump. Following $[1,21]$ the FWM efficiency, $\eta$, is defined as the ratio between the generated idler and input signal powers at the output $\left(\log _{10}\left(P_{i} / P_{s}\right)\right)$. The input signal power is measured in the absence of the pump beam with the signal tuned away from the ring resonance, therefore only taking into account the propagation losses in the bus waveguide and output chip-fibre coupling loss, which are identical for the idler. The idler output power is measured at the output of the chip. First, the FWM efficiency was measured whilst varying the ring cross-coupling coefficient as shown in Fig.5(a). The alignment of the pump and signal wavelengths to the ring resonances was optimised for each measurement point. The solid line in the plot is a calculation of the expected efficiency given the measured ring parameters following the model from [1]:

$$
\begin{array}{r}
\eta \equiv \frac{I_{i}^{(\text {out })}}{I_{s}^{(\text {in })}}=\left|\gamma P_{p} L_{\text {eff }}\right|^{2} F_{p}^{2} F_{s} F_{i} \\
L_{\text {eff }}^{2}=L^{2} \exp (-\alpha L)\left|\frac{1-\exp (-\alpha L+j \Delta k L)}{\alpha L-j \Delta k L}\right|^{2} \\
F_{p, s, i}=\left|\frac{r}{1-\operatorname{texp}\left(-\frac{-\alpha L}{2}+j k_{p, s, i} L\right)}\right|^{2}
\end{array}
$$

where the non-linear coefficient $\gamma=320(\mathrm{Wm})^{-1}$ and $\alpha$ is the average field propagation loss coefficient, taking into account both two-photon and free carrier loss terms. $L$ and $L_{e f f}$ are the cavity geometric and effective lengths respectively. $F_{p, s, i}$ is the square of the field enhancement factor for the pump, signal or idler beams, and $k_{p, s, i}$ are the wavenumbers associated with the pump, idler and signal beams. The mismatch between the wavenumbers is given by $\Delta k=2 k_{p}-k_{s}-k_{i} \cdot r$ and $t$ are the ring field cross-coupling and transmission coefficients, respectively, where $|r|^{2}=\kappa=1-|t|^{2}$. For the as-fabricated case the device exhibits a FWM efficiency of $-40 d B$, while at peak efficiency this value is increased to $-16.3 d B$, an enhancement of nearly $25 \mathrm{~dB}$. After the maximum point the efficiency drops off significantly as predicted, falling by over $10 \mathrm{~dB}$ for a change of only 0.015 in $\kappa$, highlighting the crucial role of the coupling coefficient in resonator design and its potential sensitivity to fabrication variation. Finally, the pump wavelength was detuned to longer wavelengths with respect to the signal, corresponding to more widely spaced ring resonances between the two. The limit on the spacing of the two signals was defined by the tunable laser source. A detuning bandwidth of over $50 \mathrm{~nm}$ was achieved with relatively consistent FWM efficiency, as shown in Fig.5(b).

In conclusion, given fabrication tolerances that can shift a resonator cross-coupling coefficient by a few percent, it is clear that designing devices for non-linear parametric processes at their optimal efficiency point requires active tuning of the device post-fabrication. 


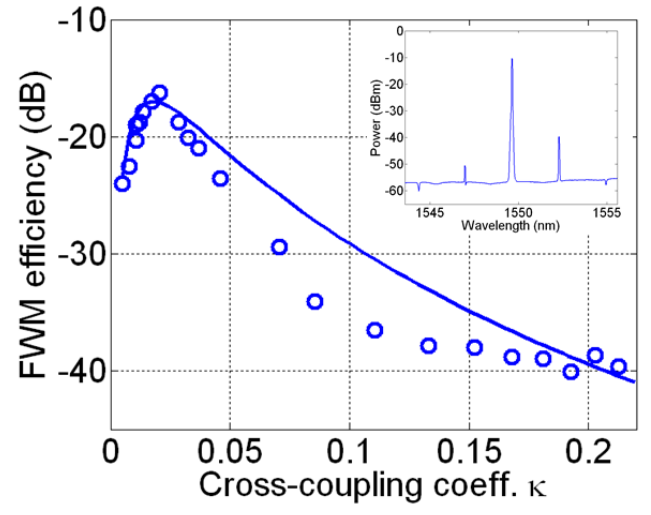

(a)

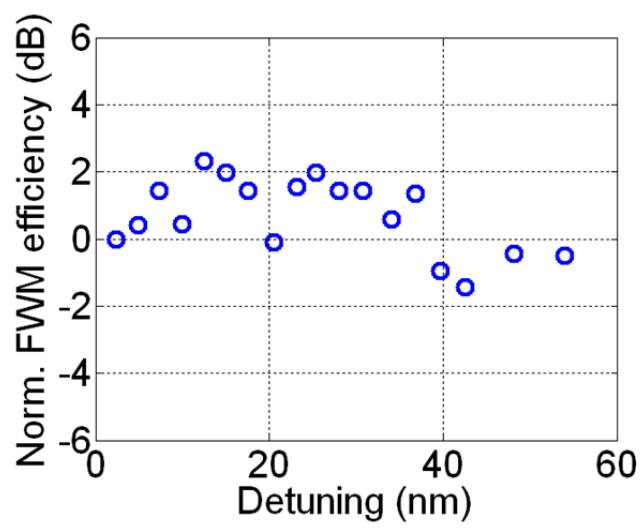

(b)

Fig. 5. (a) Measured optical FWM efficiency as a function of power dissipated on the tunable coupler, for $P_{p}=0.7 \mathrm{~mW}$. The solid line shows the theoretical model and the open circles are measured data. (Inset: measured FWM spectrum at critical coupling point) (b) Wavelength dependent FWM efficiency, normalised to the case where the pump and signal are tuned to adjacent ring resonances.

By simple electrical control of a ring resonator coupling coefficient using an asymmetrically placed resistive heating element, $\kappa$ can be tuned from 0.22 to less than 0.005 , and therefore over the critical coupling point of the ring. Record FWM efficiencies of $-16.3 d B$ were demonstrated at sub-mW power levels using the tunable ring device. In order to further increase the FWM efficiency, when operating at higher pump powers, a p-n junction could be added to the resonator in order to sweep out generated carriers and lower the non-linear losses of the system.

The authors wish to thank the staff of the James Watt Nanofabrication Center at the University of Glasgow. This work was partially supported by the EPSRC under the Cornerstone project (EP/L021129/1) and the FP-7 BBOI project (323734). M. Sorel acknowledges partial support from a CDE Precision Timing and Navigation grant.

\section{References}

1. A. C. Turner, M. A. Foster, A. L. Gaeta, and M. Lipson, Opt. Express, 16(7), 48817, (2008).

2. C. Lacava, M. J. Strain, P. Minzioni, I. Cristiani, and M. Sorel, Opt. Express, 21(18), 2158721595, (2013).

3. V. R. Almeida, C. A. Barrios, R. R. Panepucci, and M. Lipson, Nature, 431, 10811084, (2004).

4. A. Biberman, B. G. Lee, A. C. Turner-Foster, M. A. Foster, M. Lipson, A. L. Gaeta, and K. Bergman, Opt. Express, 18(17), 1804755, (2010).

5. S. Azzini, D. Grassani, M. J. Strain, M. Sorel, L. G. Helt, J. E. Sipe, M. Liscidini, M. Galli, and D. Bajoni, Opt. Express, 20(21), 2310023107, (2012).

6. Y. Guo, W. Zhang, S. Dong, Y. Huang, and J. Peng, Opt. Lett., 30(8), 2526, (2014).

7. J. R. Ong, R. Kumar, and S. Mookherjea, Opt. Lett., 39(15), 4439, (2014).

8. A. D. Bristow, N. Rotenberg, and H. M. van Driel, Appl. Phys. Lett., 90(19), 191104, (2007).

9. N. Matsuda, T. Kato, K.-I. Harada, H. Takesue, E. Kuramochi, H. Taniyama, and M. Notomi, Opt. Express, 19(21), 1986174, (2011).

10. W. Bogaerts, P. De Heyn, T. Van Vaerenbergh, K. De Vos, S. Kumar Selvaraja, T. Claes, P. Dumon, P. Bienstman, D. Van Thourhout, and R. Baets, Laser Photonics Rev., 6(1), 4773, (2012).

11. A. Parini, G. Bellanca, A. Annoni, F. Morichetti, A. Melloni, M. J. Strain, M. Sorel, M. Gay, C. Pareige, L. Bramerie, and M. Thual, IEEE Photonics Technol. Lett., 25(23), 22852288, (2013).

12. K. De Vos, I. Bartolozzi, E. Schacht, P. Bienstman, and R. Baets, Opt. Express, 15(12), 76105, (2007).

13. S. Azzini, D. Grassani, M. Galli, L. C. Andreani, M. Sorel, M. J. Strain, L. G. Helt, J. E. Sipe, M. Liscidini, and D. Bajoni, Opt. Lett., 37(18), p38079, (2012).

14. A. Yariv, IEEE Photonics Technol. Lett., 14(4), pp. 483485, (2002).

15. M. Ferrera, D. Duchesne, L. Razzari, M. Peccianti, R. Morandotti, P. Cheben, S. Janz, D.X. Xu, B. E. Little, S. Chu, and D. J. Moss, Opt. Express, 17(16), 14098103, (2009).

16. M.-C. M. Lee and M. C. Wu, Opt. Express, 1411, pp. 470312, (2006).

17. L. Wang, X. Han, Y. Gu, Y. Wang, P. Zou, J. Wang, X. Jian, G. Morthier, and M. Zhao, Opt. Commun., vol. 298299, pp. 95100, (2013).

18. P. Orlandi, F. Morichetti, M. J. Strain, M. Sorel, A. Melloni, and P. Bassi, Tunable silicon photonics directional coupler driven by a transverse temperature gradient., Opt. Lett., 38(6), 8635, (2013).

19. D. Hofstetter and R. L. Thornton, Opt. Lett., 22(24), 18313, (1997).

20. J. Poon, J. Scheuer, S. Mookherjea, G. Paloczi, Y. Huang, and A. Yariv, Opt. Express, 12(1), 90103, (2004).

21. P. P. Absil, J. V Hryniewicz, B. E. Little, P. S. Cho, R. a Wilson, L. G. Joneckis, and P. T. Ho, Opt. Lett., 25, (8), 5546, (2000). 


\section{References}

1. A. C. Turner, M. A. Foster, A. L. Gaeta, and M. Lipson, Ultra-low power parametric frequency conversion in a silicon micro-ring resonator., Opt. Express, 16(7), 48817, (2008).

2. C. Lacava, M. J. Strain, P. Minzioni, I. Cristiani, and M. Sorel, Integrated nonlinear Mach Zehnder for 40 Gbit / s all-optical switching, Opt. Express, 21(18), 2158721595, (2013)

3. V. R. Almeida, C. A. Barrios, R. R. Panepucci, and M. Lipson, All-optical control of light on a silicon chip, Nature, 431, 10811084, (2004).

4. A. Biberman, B. G. Lee, A. C. Turner-Foster, M. A. Foster, M. Lipson, A. L. Gaeta, and K. Bergman, Wavelength multicasting in silicon photonic nanowires., Opt. Express, 18(17), 1804755, (2010).

5. S. Azzini, D. Grassani, M. J. Strain, M. Sorel, L. G. Helt, J. E. Sipe, M. Liscidini, M. Galli, and D. Bajoni, Ultralow power generation of twin photons in a compact silicon ring resonator, Opt. Express, 20(21), 2310023107, (2012).

6. Y. Guo, W. Zhang, S. Dong, Y. Huang, and J. Peng, Telecom-band degenerate-frequency photon pair generation in silicon micro-ring cavities, Opt. Lett., 30(8), 2526, (2014).

7. J. R. Ong, R. Kumar, and S. Mookherjea, Silicon microring-based wavelength converter with integrated pump and signal suppression., Opt. Lett., 39(15), 4439, (2014).

8. A. D. Bristow, N. Rotenberg, and H. M. van Driel, Twophoton absorption and Kerr coefficients of silicon for 8502200 nm, Appl. Phys. Lett., 90(19), 191104, (2007).

9. N. Matsuda, T. Kato, K.-I. Harada, H. Takesue, E. Kuramochi, H. Taniyama, and M. Notomi, Slow light enhanced optical nonlinearity in a silicon photonic crystal coupled-resonator optical waveguide., Opt. Express, 19(21), 1986174, (2011).

10. W. Bogaerts, P. De Heyn, T. Van Vaerenbergh, K. De Vos, S. Kumar Selvaraja, T. Claes, P. Dumon, P. Bienstman, D. Van Thourhout, and R. Baets, Silicon micro-ring resonators, Laser Photonics Rev., 6(1), 4773, (2012).

11. A. Parini, G. Bellanca, A. Annoni, F. Morichetti, A. Melloni, M. J. Strain, M. Sorel, M. Gay, C. Pareige, L. Bramerie, and M. Thual, BER Evaluation of a Passive SOI WDM Router, IEEE Photonics Technol. Lett., 25(23), 22852288, (2013).

12. K. De Vos, I. Bartolozzi, E. Schacht, P. Bienstman, and R. Baets, Silicon-on-Insulator micro-ring resonator for sensitive and label-free biosensing., Opt. Express, 15(12), 76105, (2007).

13. S. Azzini, D. Grassani, M. Galli, L. C. Andreani, M. Sorel, M. J. Strain, L. G. Helt, J. E. Sipe, M. Liscidini, and D. Bajoni, From classical four-wave mixing to parametric fluorescence in silicon micro-ring resonators., Opt. Lett., 37(18), p38079, (2012).

14. A. Yariv, Critical coupling and its control in optical waveguide-ring resonator systems, IEEE Photonics Technol. Lett., 14(4), pp. 483485, (2002).

15. M. Ferrera, D. Duchesne, L. Razzari, M. Peccianti, R. Morandotti, P. Cheben, S. Janz, D.X. Xu, B. E. Little, S. $\mathrm{Chu}$, and D. J. Moss, Low power four wave mixing in an integrated, micro-ring resonator with $\mathrm{Q}=1.2$ million.,
Opt. Express, 17(16), 14098103, (2009).

16. M.-C. M. Lee and M. C. Wu, Tunable coupling regimes of silicon microdisk resonators using MEMS actuators., Opt. Express, 1411, pp. 470312, (2006).

17. L. Wang, X. Han, Y. Gu, Y. Wang, P. Zou, J. Wang, X. Jian, G. Morthier, and M. Zhao, A tunable polymer waveguide ring filter fabricated with UV-based soft imprint technique, Opt. Commun., vol. 298299, pp. 95100, (2013).

18. P. Orlandi, F. Morichetti, M. J. Strain, M. Sorel, A. Melloni, and P. Bassi, Tunable silicon photonics directional coupler driven by a transverse temperature gradient., Opt. Lett., 38(6), 8635, (2013).

19. D. Hofstetter and R. L. Thornton, Theory of loss measurements of Fabry Perot resonators by Fourier analysis of the transmission spectra., Opt. Lett., 22(24), 18313, (1997).

20. J. Poon, J. Scheuer, S. Mookherjea, G. Paloczi, Y. Huang, and A. Yariv, Matrix analysis of micro-ring coupled-resonator optical waveguides., Opt. Express, 12(1), 90103, (2004).

21. P. P. Absil, J. V Hryniewicz, B. E. Little, P. S. Cho, R. a Wilson, L. G. Joneckis, and P. T. Ho, Wavelength conversion in GaAs micro-ring resonators., Opt. Lett., $25,(8), 5546,(2000)$. 